\title{
REGULAR AND CHAO'TIC BEHAVIOUR OF A KICKED DAMPED SPIN
}

\author{
J.A. Holyst* AND A. SUkIEnNICKI \\ Institute of Physics, Warsaw Technical University \\ Koszykowa 75, 00-662 Warszawa, Poland
}

(Received October 31, 1991)

The dynamics of a kicked, anisotropic, damped spin is reduced to a two-dimensional map. This map exhibits such features as bifurcation diagrams, regular or chaotic attractors/repellors and intermittent-like transitions between two strange attractors. With increase of damping a transition from chaos to the fixed point attractor occurs. On the contrary to the Hamiltonian case the type of magnetic anisotropy plays a crucial role for damped models.

PACS numbers: $05.45 .+\mathrm{b}, 75.10 . \mathrm{Hk}$

The evidence of a deterministic chaos [1-2] appearing in spin models and magnetic materials was reported in several theoretical [3-4] and experimental papers [5-6]. However, although real magnets do suffer effects of dissipation, up to now the damping was included only in theoretical investigations on chaotic motion of domain walls [7-8]. The aim of this paper is to study effects of dissipation on the simple but (as we think) important model of a kicked, anisotropic spin. Preliminary results were published in the paper [9].

Let us consider a single classical spin $S(t),(|S|=S)$ in the uniaxial anisotropy field and in the presence of the transversal, time-dependent magnetic field $\tilde{B}(t)$. The Iramiltonian of the system has the form

$$
H=-A\left(S^{z}\right)^{2}-\tilde{B}(t) S^{x} .
$$

Here, the presence of the perpendicular field $\tilde{B}(t)$ directed along the $x$-axis breaks the uniaxial, Ising-like $(A>0$ favours easy $z$-axis) or planar $(A<0$ favours easy $x y$-plane) symmetry connected with a magnetic anisotropy $A$. The above Hamiltonian well describes a ferromagnetic single-domain probe analysed recently from the point of view of macroscopic quantum phenomena [10] or a magnetic ion in a paramagnetic material [11].

*A part of this work has been performed during a stay at the Institut für Theoretische Physik, Universität Hannover, Appelstrasse 2, 3000 Hannover 1, Germany 
The time evolution of this system can be described by the Landau-Lifshitz equation of motion with the damping term

$$
\mathrm{d}_{t} S=S \times B_{\mathrm{eft}}-\lambda S \times\left(S \times B_{\mathrm{eff}}\right) / S,
$$

where $\boldsymbol{B}_{\text {eff }}=-\mathrm{d} H / \mathrm{d} \boldsymbol{S}$ is the effective magnetic field acting on the spin $S$ and $\lambda$ $(\lambda \geq 0)$ is the damping parameter. This type of damping is frequently used in the theory of dynamics of magnetic systems [7-8, 11-12] based on the micromagnetic approach and a connection of this damping with the more general formulation of relaxation mechanism in many body systems can be found in the Ref. [13]. The dissipative character of the second term on the right hand side of Eq. (2) can be easily seen if one notices that a total time derivative of a IIamiltonian can be written in spherical coordinates as

$$
\frac{\mathrm{d} H}{\mathrm{~d} t}=-\frac{\lambda}{S}\left[\left(\frac{\partial H}{\partial \vartheta}\right)^{2}+\frac{1}{\sin ^{2} \vartheta}\left(\frac{\partial H}{\partial \varphi}\right)^{2}\right]+\frac{\partial H}{\partial t} .
$$

From (3a) it follows that for $\partial H / \partial t=0$ there is $\mathrm{d} H / \mathrm{d} t<0$ due to the positivity of $\lambda$. This fact does not mean however that a flow in the phase space always contracts. Calculating from Eq. (2) a rate of contraction of the $(\varphi, p)$ phase space (where $p$ denotes $S \cos \vartheta)$ one gets

$$
\frac{\partial \dot{\varphi}}{\partial \varphi}+\frac{\partial \dot{p}}{\partial p}=\frac{-\lambda}{S}\left(\partial_{\vartheta \vartheta}^{2} H+\cot \vartheta \partial_{\vartheta} H+\sin ^{-2} \vartheta \partial_{\varphi \varphi}^{2} H\right) .
$$

It follows from (3b) that in general this rate is not equal to a constant and it can be positive or negative depending on the point in the phase space and model parameters (it corresponds to repelling or attracting regions in the phase space respectively).

In order to simplify calculations we assume that the magnetic field $\tilde{B}(t)$ in the Hamiltonian (1) is a periodic series of delta-like pulses with period $\tau$

$$
\tilde{B}(t)=B \sum_{n=1}^{\infty} \delta(t-n \tau) \text {. }
$$

The time evolution of the spin $S(t)$ during each period of the field can be divided into two parts: in the absence of the magnetic field and in the presence of this field. For the first case, e.g. for $t \in\left(0_{+}, \tau+0_{-}\right)$one gets from $\mathrm{Eq}$. (2) a map $T_{A}$ describing the evolution of the spin between two subsequent pulses of the field $T_{A}\left[S\left(t=0_{+}\right)\right]=S\left(t=\tau+0_{-}\right)$. This map can be written as follows:

$$
\begin{aligned}
& S^{x} \rightarrow S^{x^{\prime}}=c W\left[S^{x} \cos (\Delta \varphi)-S^{y} \sin (\Delta \varphi)\right], \\
& S^{y} \rightarrow S^{y^{\prime}}=c W\left[S^{y} \cos (\Delta \varphi)+S^{x} \sin (\Delta \varphi)\right], \\
& S^{z} \rightarrow S^{z^{\prime}}=W S^{z},
\end{aligned}
$$

where $c=\exp (-2 \lambda A S \tau), W=\left[c^{2}+\left(S^{z} / S\right)^{2}\left(1-c^{2}\right)\right]^{-1 / 2}$ and $\Delta \varphi=\lambda^{-1} \ln \{[1+$ $\left.\left.\left(S / S^{z}\right)\right] /\left[1+\left(S / S^{z^{\prime}}\right)\right]\right\}-2 A S \tau$. The Jacobian of this map is equal to $J_{A}=c^{2} W^{3}$.

The effect of the pulse of the magnetic field can be written as the following $\operatorname{map} T_{B}\left[S\left(n \tau+0_{-}\right)\right]=S\left(n \tau+0_{+}\right)$

$$
S^{x} \rightarrow S^{x^{\prime}}=S-2 S D^{2}\left(S-S^{x}\right) U,
$$




$$
\begin{aligned}
& S^{y} \rightarrow S^{y^{\prime}}=2 D S U\left(S^{y} \cos B+S^{z} \sin B\right), \\
& S^{z} \rightarrow S^{z^{\prime}}=2 D S U\left(S^{z} \cos B-S^{y} \sin B\right),
\end{aligned}
$$

where $D=\exp (-\lambda B)$ and $U=\left[S+S^{x}+D^{2}\left(S-S^{x}\right)\right]^{-1}$. The Jacobian of this map is $J_{B}=(2 S D U)^{2}$. Combining the expressions (5) and (6) we get

$$
S\left(n \tau+0_{-}\right)=T_{A}\left\{T_{B}\left[S\left((n-1) \tau+0_{-}\right)\right]\right\}=T\left[S\left((n-1) \tau+0_{-}\right)\right]
$$

or simply $S_{n}=T\left(S_{n-1}\right)$. It is important to note that due to a constraint $|S|=S$, Eqs. $(5 \mathrm{a}-\mathrm{c})$ and $(6 \mathrm{a}-\mathrm{c})$ represent two-dimensional, invertible map. The limit $(\lambda \rightarrow 0)$ of the map $T$ corresponds exactly to the map used in the Refs. [3-4].

It is easy to check that the points $\left(S^{x}= \pm S, S^{y}=S^{z}=0\right)$ are two trivial fixed points of the map (7). In order to check the stability of these points we linearized the map (7) around the point $\left(S^{x}=S, S^{y}=S^{z}=0\right)$ (the stability analysis of the second trivial fixed point is performed in the similar way changing $B$ to $-B$ ) and we found that for $\lambda \ll 1$ eigenvalues of the linearized two-dimensional map are

$$
\begin{aligned}
& \Lambda_{1(2)}=(1+\lambda A S \tau-\lambda B)\{A S \tau \sin B+\cos B \\
& \left. \pm\left[(A S \tau \sin B+\cos B)^{2}-1\right]^{1 / 2}\right\} .
\end{aligned}
$$

It follows from (8) that for $\lambda=0$ the system is stable provided Eq. (9a) (for $A>0$ ) or $\mathrm{Eq} .(9 \mathrm{~b})$ (for $A<0$ ) is fulfilled,

$$
\begin{array}{llll}
0 \geq \tan (B / 2) \geq-(A S \tau)^{-1} & \text { or } & & \tan (B / 2) \geq A S \tau \\
0 \leq \tan (B / 2) \leq-(A S \tau)^{-1} & \text { or } & & \tan (B / 2) \leq A S \tau
\end{array}
$$

On the other hand it follows from Eq. (8) that for small but non-zero damping $\lambda \ll 1$ the values of magnetic fields given by Eqs. (9) correspond to the stable fixed point provided that the field is larger than some critical value $B_{\mathrm{c}}=A S \tau$. It is interesting that for $B<B_{\mathrm{c}}$, the non-zero damping causes the increase of the modulus of eigenvalues $A_{1(2)}$ with respect to the undamped case.

The map (7) was investigated by us numerically and without any loss of generality we assumed $S=1$ and $\tau=2 \pi$. For $\lambda=0$ three types of solutions are possible [3-4]: regular solutions (case a), regular solutions surrounded by a chaotic sea (case b - see Fig. 1) and a chaotic sea filling the whole phase space (case c). We observed that for the values of $A$ and $B$ corresponding to the cases (a)-(b) and for non-zero damping the transient evolution of the system towards a fixed point attractor occurred. Depending on the initial conditions various types of the transients were found. A spiral transient occurred for the initial condition chosen from the regular region of the phase space containing the attractor. If the starting point was inside the chaotic sea the transient filled this sea more or less densely, then jumped suddenly to one of the regular regions surrounding a corresponding fixed point and next reached this point by a spiral. As the regular region or the chaotic sea we understand here the respective parts of the phase space in the case $\lambda=0$ (Fig. 1). If the initial point was chosen inside the regular region surrounding 

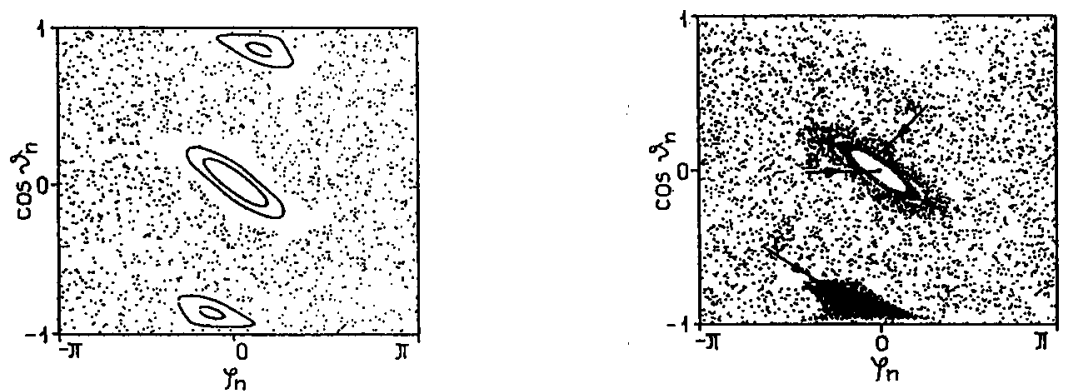

Fig. 1. Seven various trajectories of the map (13) for $A=0.5, B=-0.5$ and $\lambda=0$. Each trajectory consists of 3000 iterations.

Fig. 2. A single trajectory (a very long transient) for $A=0.5, B=-0.5, \lambda=10^{-4}$ and $10^{4}$ iterations. Notice the influence of damping (compare Fig. 1). The trajectory starts in the point A near the repellor $B(\varphi=0, \cos \vartheta=0)$ then fills the chaotic sea and it ends in the point attractor $C$.

a repelling point the transient consisted of an unwinding spiral, a random walk over the chaotic sea and a sudden jump to a spiral reaching the fixed point (Fig. 2). The bigger the parameter $\lambda$, the earlier the fixed point is reached (for the same starting points). Our results in this point are similar to those of Ref. [14] where a capture of stochastic trajectories under the influence of small dissipation in a model of a nonlinear oscillator was observed.

For the case (c) if a non-zero damping was included a very interesting transition from the regular behaviour for larger values of $\lambda$ to the chaotic behaviour for smaller values of $\lambda$ was obtained. Two cases were investigated systematically: (i) the case of Ising-like anisotropy with $A=B=1$ and (ii) the case of planar. anisotropy with $A=-1, B=1$. The projections of the resulting attractors in $(\varphi, \cos \vartheta)$ phase space onto $\varphi$ axis are shown in Figs. $3 \mathrm{a}-\mathrm{b}$.

For the case (i) of the Ising-like symmetry three characteristic values of the parameter $\lambda$ can be seen: $\lambda_{1}=0.1618, \lambda_{c}=0.1172285$ and $\lambda_{i}=0.1054942$. For all values of $\lambda$ bigger than $\lambda_{1}$ the system evolves, depending on the initial conditions, to one of two point-like attractors where both of them are symmetric with respect to each other, i.e. with respect to a transformation $\cos \vartheta \rightarrow-\cos \vartheta$, $\varphi \rightarrow-\varphi$. At the value $\lambda_{1}$ the first period-doubling bifurcation appears. A sequence of next period doubling bifurcations terminates at $\lambda_{c}$ and for $\lambda<\lambda_{c}$ the motion is chaotic (although some regular windows appear). The values of the first five ratios $\delta_{k}=\left(\lambda_{k+1}-\lambda_{k}\right) /\left(\lambda_{k+2}-\lambda_{k+1}\right)$ are the following: $14.82,3.62,4.50,4.60$, and one finds a good convergence to $\delta=4.67$ which is the characteristic Feigenbaum value for dissipative systems with quadratic extremum maps. This result should not be mistaken with the studies on scaling of Feigenbaum constants during a crosssover between dissipative and hamiltonian dynamics in the Henon map [15] because in our case the damping parameter $\lambda$ is simultaneously the chaos control parameter.

Two symmetric (simple or strange) attractors exist for $\lambda>\lambda_{\mathrm{i}}$. For $\lambda=\lambda_{\mathrm{i}}$. however the coalescence of both attractors occurs and for $\lambda<\lambda_{i}$ the spin starts to move between both of them (see Fig. 4a). For $\lambda<\lambda_{i}$, as the paraneter $\lambda$ is made 

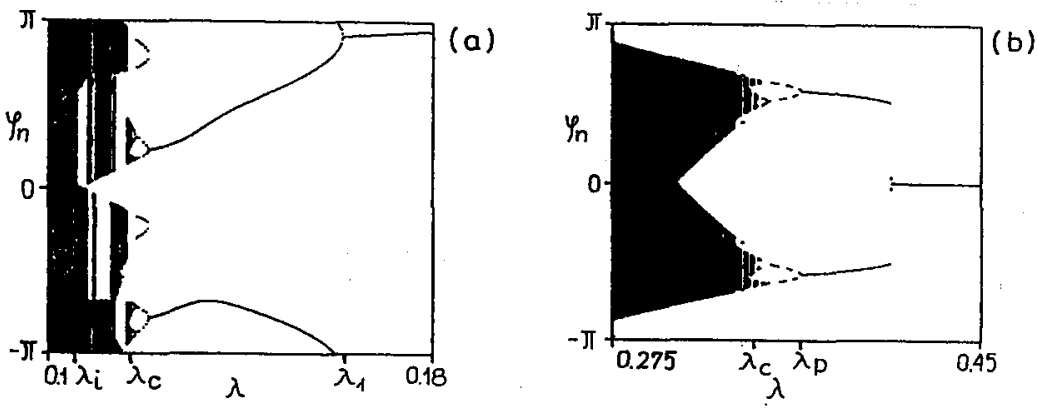

Fig. 3. Projections of the attractors on $\varphi$ axis for $A=B=1$ (a) and $A=-1, B=1$ (b) as a function of a damping parameter $\lambda$. The starting point was fixed as $\varphi_{0}=0.1$ and $\cos \vartheta_{0}=0.1$. The broken character of attractors legs is connected with crossing by this point a boundary between distinct basins of attractors as we varied the values of the parameter $\lambda$. In Fig. $3 \mathrm{~b}$ besides the period doubling bifurcations we observed $\mathrm{a}$ pitchfork bifurcation at $\lambda_{\mathrm{p}}=0.365$.
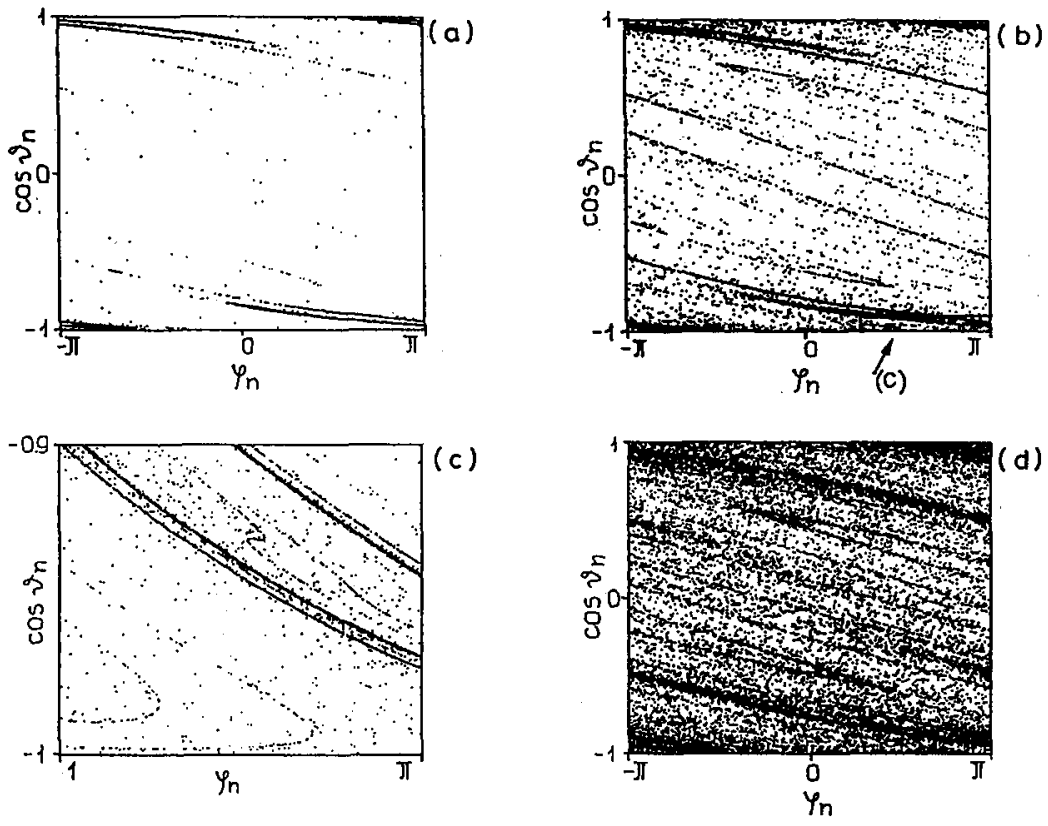

Fig. 4. Strange attractors obtained for $A=B=1, \lambda=0.1054$ (a); $\lambda=0.1$ (b-c); $\lambda=0.05(\mathrm{~d})$.

smaller and smaller, then the time of stay of the system on the lower or upper branch of the attractor is shorter and shorter and a rising number of scattered points in the middle region of the phase space is observed. Due to this fact the separation between the two branches of the attractor becomes less and less evident (Figs. 4a-d). 
Apart from strange attractors, our model exhibits also another interesting feature, i.e. strange repellors [16-17]. In Fig. 5 we present a strange repellor of the map $T$ obtained as an attractor of the map $T^{-1}=T_{-B} T_{-A}$.

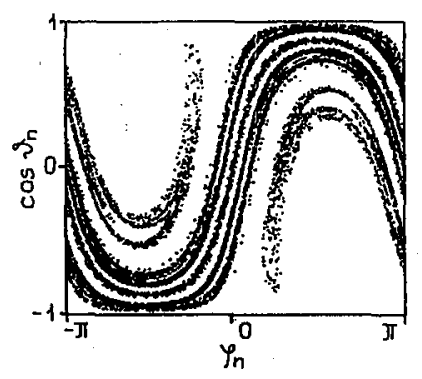

Fig. 5. A strange repellor of the map $T$ for $A=1, B=1, \lambda=0.1054$.

Moreover, for values of $\lambda$ lover but comparable to $\lambda_{i}$ the motion has the intermittent-like character shown in Fig. 6 and the upper or lower branch of the attractor corresponds to a laminar region in the standard theory of the intermittency $[2,18]$. In the limit $\lambda \rightarrow \lambda_{\mathrm{i}^{-}}$the mean length of this "laminar region"
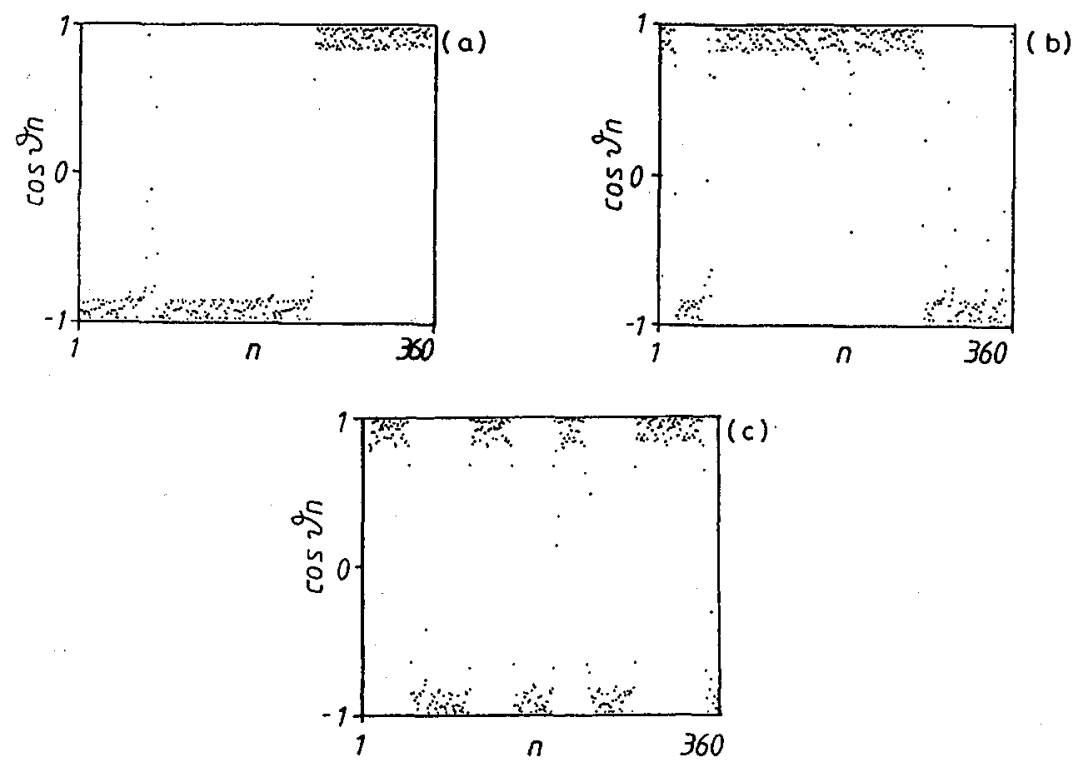

Fig. 6. An intermittent-like evolution of the variable $\cos \vartheta_{n}$ for $A=B=1$ and $\lambda=0.105(\mathrm{a}) ; \lambda=0.104(\mathrm{~b}) ; \lambda=0.102$ (c).

tends to infinity (we were not able however to find any scaling law describing this divergence).

The existence of an upper and a lower branch of the attractor is a consequence of the Ising-like symmetry of anisotropy. Since the $z$-axis is the easy 
magnetization axis thus the spin tends (neglecting the effects of the field) to stay in one of the favorable energetically positions so long as the damping is so high that it is impossible to jump from one to another energy well. If the damping is lower however such jumps are possible. Thus the intermittency bursts appear. They play here a similar role as the kink-solitons restoring the symmetry in the Ising-like chains [19]. The only difference is that kinks connect the different domains in space while the intermittent bursts connect the different domains in time. A similar phenomenon of merging two distinct attractors was observed first in Ref. [20] where a driven particle in a double well potential was studied.

Quite different results were obtained for the case (ii) of the planar symmetry of the anisotropy. One can notice that due to the symmetry of the equation of motion the only difference for the trajectories $\left(\varphi_{n}, \cos \vartheta_{n}\right)$ obtained with no damping for cases $A=-1$ and $A=1$ is a transformation $\varphi_{n} \rightarrow \varphi_{n}+\pi, \vartheta_{n} \rightarrow \pi-\vartheta_{n}$. However, in the presence of the small but non-zero damping (i.e. $\lambda \ll 1$ ) the positions of attractors and repellors observed by us for both these cases were nearly exactly interchanged. Thus the effect of damping is quite different for the case (ii) for $\lambda<\lambda_{c}$. One should notice for example that although with a decrease of damping the bifurcation sequence is seen as in Fig. $3 \mathrm{~b}$ but there is no point in this figure corresponding to $\lambda_{\mathrm{i}}$ of Fig. 3a. In the case (ii) there is an attractor in the centre of the phase space $(\varphi, \cos \vartheta)$ and no coalescence of two attractors occurs. This is a result of the existence of only one energy minimum at $\cos \vartheta=0, \varphi=0$ for the case (ii) instead of two degenerated energy minima corresponding to $\cos v>0$ or $<0$ for the case (i).

In both cases considered above, if we forget about the existence of narrow periodic windows, the general effect of damping is in suppressing a tendency for chaos (Figs. 3a-b).

Although some of our results (Fig. 2) resemble the previous studies on the nonlinear oscillator [14] and mode-coupling phenomena in plasma systems [21], some other features of our model are quite different. For example in the quoted models the increase of damping causes a clear transition from periodic to strange attractors while a tendency in our model was just opposite. Moreover, we observed an interesting influence of a symmetry of magnetic anisotropy on the properties of attractors. Our model seems to be more difficult to handle by analytical methods than Fenon map [15] because its Jacobian $J_{A} J_{B}$ depends on the point in the phase space. On the other hand, due to the connections of this model to real magnetic systems there is a chance that the results of our computer simulations will be verified by an experiment.

\section{Acknowledgements}

This work was supported by the Institute for Low Temperatures and Structural Researches of the Polish Academy of Sciences under Project 01.12.2.3. One of us (J.A.H.) is grateful to Alexander von Ilumboldt Foundation for a Research Fellowship and to Professor H.J. Mikeska for his hospitality during the author's stay at the University Hannover. We are thankful to Prof. II.J. Mikeska, Dr. H. Frahm and Dr. J.J. Żebrowski for helpful discussions. 


\section{References}

[1] A.J. Lichtenberg, M.A. Lieberman, Regular and Stochastic Motion, Springer, New York 1983.

[2] Universality in Chaos, Ed. P. Cvitanović, Adam Hilger, Bristol 1989.

[3] H. Frahm, H.J. Mikeska, Z. Phys. B, Condens. Matter 60, 117 (1985).

[4] K. Nakamura, Y. Okaazaki, A.R. Bishop, Phys. Rev. Lett. 57, 5 (1986); F. Haake, M. Kuś, R. Scharf; Z. Phys. B, Condens. Matter 65, 381 (1987).

[5] G. Gibson, C. Jeffries, Phys. Rev. A 29, 811 (1984).

[6] H. Benner, F. Rödelsperger, H. Seitz, G. Weise, J. Phys. (France) 49, 1603, C8 (1988).

[7] H. Suhl, X.Y. Zhang, J. Appl. Phys. 61, 4216 (1987).

[8] J.J. Żebrowski, Phys. Rev. B 39, 7205 (1989).

[9] J.A. Holyst, A. Sukiennicki, J. Magn. Magn. Mater., to be published.

[10] A. Garg, G.-H. Kim, Phys. Rev. Lett. 63, 2513 (1989).

[11] A.H. Morrish, The Physical Principles of Magnetism, John Wiley, New York 1965.

[12] J.A. Hołyst, A. Sukiennicki, J.J. Żebrowski, Phys. Rev. B 33, 3492 (1986).

[13] J.A. Hołyst, E.A. Turski, Phys. Rev. B 34, 1937 (1986).

[14] B.V. Chirikov, P.M. Izraelev, Physica D 2, 30 (1981).

[15] J.P. van der Weele, H.W. Capel, T. Post, Ch.J. Calkoen, Physica A 137, 1 (1986).

[16] H. Kantz, P. Grassberger, Physica D 17, 75 (1985).

[17] T. Tél, in: Directions in Chaos, Ed. Hao Bai-lin, Vol. 3, World Scientific, Singapore. 1990.

[18] Y. Pomeau, P. Manneville, Commun. Math. Phys. 74, 189 (1980).

[19] R. Rajaraman, Solitons and Instantons, North-Holland, Amstcrdam 1982.

[20] H. Ishii, H. Fujisaka, M. Inoue, Phys. Lett. A 116, 257 (1986).

[21] C. Meunier, M.N. Bussac, G. Laval, Physica D 4, 236 (1982). 